\title{
Is vertical migration in Antarctic krill (Euphausia superba) influenced by an underlying circadian rhythm?
}

\author{
EDWARD GATEN ${ }^{1 *}$, GERAINT TARLING $^{3}$, HAROLD DOWSE $^{4}$, CHARALAMBOS KYRIACOU $^{2}$ \\ and EZIO ROSATO ${ }^{2 *}$ \\ ${ }^{1}$ Department of Biology, University of Leicester, Leicester LE1 7RH, UK \\ ${ }^{2}$ Department of Genetics, University of Leicester, Leicester LE1 7RH, UK \\ ${ }^{3}$ British Antarctic Survey, High Cross, Madingley Rd, Cambridge CB3 OET, UK \\ ${ }^{4}$ School of Biology and Ecology, University of Maine, Orono, ME 04469, USA
}

\begin{abstract}
Antarctic krill (Euphausia superba) is a keystone species in the southern ocean ecosystem where it is the main consumer of phytoplankton and constitutes the main food item of many higher predators. Both food and predators are most abundant at the surface, thus krill hide in the depth of the ocean during the day and migrate to the upper layers at night, to feed at a time when the predatory risk is lowest. Although the functional significance of this diel vertical migration (DVM) is clear and its modulation by environmental factors has been described, the involvement of an endogenous circadian clock in this behaviour is as yet not fully resolved. We have analysed the circadian behaviour of Euphausia superba in a laboratory setting and here we present the first description of locomotor activity rhythms for this species. Our results are in agreement with the hypothesis that the circadian clock plays a key role in DVM. They also suggest that the interplay between food availability, social cues and the light:dark cycle acts as the predominant Zeitgeber for DVM in this species.
\end{abstract}

[Gaten E., Tarling G., Dowse H., Kysiacou C. and Rosato E. 2008 Is vertical mignation in Antarctic krill (Euphausia superba) influenced by an underlying circadian rhythm? J. Genet. 87, 473-483]

\section{Introduction}

Behaviour in eukaryotic organisms is profoundly influenced by diurnal and seasonal changes in their environment. The evolution of circadian clocks has allowed these organisms to regulate molecular and physiological rhythms, which in turn affect the animals' rhythmic behaviour.

Many components of the plankton exhibit diel vertical migration (DVM), usually rising towards the surface at night and moving to deeper water during the day. In this way, the more abundant food resources present nearer to the surface can be exploited at night and visually oriented predators can be avoided by day (Gliwicz 1986). Although several exogenous factors have been shown to influence vertical migration, it is generally assumed that light is the most significant of these, determined either by the absolute light intensity or

*For correspondence. E-mail: gat@le.ac.uk; er6@1e.ac.uk. by the rate of intensity change (Forward 1988; Ringelberg 1995). The underlying mechanisms behind this behaviour have not been resolved, but it seems likely that an endogenous circadian clock is involved.

In krill (Euphausiidae), extensive vertical migration often takes place, although there is considerable variation between species in the amplitude of the migration. In Atlantic waters, northern krill, Meganyctiphanes norvegica, undertake DVM of up to $800 \mathrm{~m}$ (Tarling et al. 1999). Euphausia pacifica, in the Pacific Ocean, migrates an average distance of around $200 \mathrm{~m}$ to reach the surface layers at night (Youngbluth 1975). In the southern ocean, Euphausia frigida, Thysanoessa vicina and T. macrura all migrate from $100 \mathrm{~m}$ to the surface at night, while $E$. triacantha makes a diel migration in the order to 250 to $500 \mathrm{~m}$ (Mauchline and Fisher 1969).

The situation in Antarctic krill (E. superba) is not so clear. Vertical migration in this species is reduced in amplitude (no more than $100 \mathrm{~m}$ ) and variable in extent (Godlewska

Keywords. Antarctic krill; vertical migration; circadian rhythm. 
1996). A further factor is that Antarctic krill are believed to disperse during night-time and to reform into tightly packed swarms during the daytime (Everson 1982, 1983) making it difficult to detect vertical migration of the mean population using nets or acoustics. In some instances, the krill may even migrate to the very surface, out of the range of traditional sampling technologies.

One major issue in polar environments is the extreme changes in environmental conditions through the season. Large areas of open waters become covered with ice during the winter, phytoplankton rapidly blooms and then disappears, and the light cycle moves from continuous light to continuous darkness over the course of the year. In such changing conditions, polar organisms must continue to maintain essential physiological processes. Therefore, although Antarctic krill may appear to have very few predictable patterns at the population level, individuals may maintain rhythms as a means of balancing the need to feed with that to take refuge, digest and maintain essential metabolic functions. Cottier et al. (2006) for instance showed indirect evidence of individual copepods regularly migrating up and down during midnight sun conditions in the Arctic even though there was little detectable vertical movement of the population as a whole. These individuals were alternating between phases of high and low activity at a rhythm as much set by their own internal clock as by exogenous cues. Such behaviour appears particularly suited to environments where important external cues, such as the rate of change of light, are periodically absent.

Antarctic krill face similar conditions in terms of the variability of their environment and periodic absence of external cues. We hypothesise that underlying circadian rhythms play an important role in the behaviour of these organisms. This is reinforced by the finding that even a nonpolar Euphausiid, northern krill, exhibits a quasi-diel periodicity in patterns of activity in the absence of an external light:dark cycle (Velsch and Champalbert 1994). In the current study, we have attempted to characterize vertical migration in Antarctic krill using a modification of the activity monitor of Velsch and Champalbert (1994).

\section{Materials and methods}

\section{Specimen capture}

Antarctic krill were caught during January and February 2008 (Antarctic summer) during the Discovery 2010 cruise JR177 aboard the RRV 'James Clark Ross'. Krill from two areas were used, from around $60^{\circ} \mathrm{S}$ from the vicinity of the South Orkney Islands (experiments JCR2 and JCR3), and from north-west of South Georgia at around $52^{\circ} \mathrm{S}$ (experiment JCR4). All of the krill were taken by target fishing, mainly using a pelagic net (RMT8), based on observations of krill swarms using an EK60 echo sounder at 38 and 120 $\mathrm{KHz}$. The nets were towed for only a short time and, after hauling, the krill were transferred as quickly as possible to the cold room at $1-3^{\circ} \mathrm{C}$ for sorting. Light levels in the cold room were less than $5 \mu \mathrm{mol} \mathrm{m} \mathrm{m}^{-2} \mathrm{~s}^{-1}$ and the animals were transferred to the activity monitor within $10 \mathrm{~min}$.

\section{Activity monitor}

The activity monitor is a purpose-built apparatus comprising 12 vertical tubes made of acrylic resin, painted matt black except for a transparent lid. Each tube contains 51 of seawater and is retained upright in a light-tight box. The tubes are $48 \mathrm{~cm}$ in height with a diameter of $15 \mathrm{~cm}$ and each has infra-red barriers $5 \mathrm{~cm}$ from the top and bottom of the tube. The output from these barriers is recorded continuously via a datalogger to a laptop computer running Spike2 software (Cambridge Electronic Design, Cambridge, UK). The lighting within the apparatus is adjusted to that experienced by the krill at normal daytime depths (ca. $50 \mathrm{~m}$ ) and the temperature in the cool-room kept at the level of the seawater supply $\left(1-3^{\circ} \mathrm{C}\right)$. Krill are loaded individually into 11 of the tubes containing filtered seawater, with the other tube containing a datalogger recording the light and temperature in the tube throughout the experiment. The animals were not fed during the experiments as changes in food abundance have a direct effect on locomotor activity (Fernández de Miguel and Aréchiga 1994). The computer records the number of times that the krill break the infra-red beams over a period of up to 10 days. The krill spend the first five days under a light/dark cycle (LD) close to that of the region from which they were taken in order to record their activity patterns under normal lighting conditions. This is followed by up to five days in total darkness (DD) to see whether any observed activity pattern is maintained under constant conditions.

Three experiments were carried out (table 1), using 11 animals during the first two runs and eight during the final run. The first two runs consisted of five days in LD followed by three days in DD and the third run consisted of five days in LD followed by five days in DD. Mortality rates varied between the experiments, with 9 out of 11 surviving the first experiment, 5 out of 11 in the second and 7 out of 8 in the third. The difference in mortality is thought to be primarily due to the condition of the animals at the start of the experiment. The mean lengths of the animals used were $43.8 \mathrm{~mm}$ (s.d. 2.6) in experiment 1, $51.2 \mathrm{~mm}$ (s.d. 2.3) in experiment 2 and $51.7 \mathrm{~mm}$ (s.d. 3.7) in experiment 3. All of the animals were in intermoult, except two of the animals in the final experiment, which were in premoult.

\section{Data analysis}

Data for each animal were sorted into $30 \mathrm{~min}$ bins, separated into LD and DD segments, and the resulting time series tested for periodicity using CLEAN (Roberts et al. 1987), maximum entropy spectral analysis (MESA) and autocorrelation (Levine et al. 2002a). Autocorrelation is a standard and highly robust method for detecting significant periodicities in data (Chatfield 1989). MESA is a sensitive, 
Diel vertical migration in Antarctic krill

Table 1a. Environmental parameters for each experimental sample. Ambient conditions at time of capture.

\begin{tabular}{lcccccccc}
\hline $\begin{array}{l}\text { Experiment } \\
\text { (date) }\end{array}$ & Latitude & $\begin{array}{l}\text { Time of } \\
\text { capture }\end{array}$ & $\begin{array}{l}\text { Depth } \\
\text { range }\end{array}$ & Sunrise & Sunset & Moonrise & Moonset & Civil twilight \\
\hline JCR2 (05.01.08) & $60.44^{\circ} \mathrm{S}$ & 14.00 & $17-65 \mathrm{~m}$ & 2.56 & 21.37 & $23.59^{1,2}$ & 20.39 & $1.26-23.05$ \\
JCR3 (16.01.08) & $59.66^{\circ} \mathrm{S}$ & 15.45 & $60-63 \mathrm{~m}$ & 3.11 & 20.59 & $13.09^{3}$ & 22.07 & $1.55-22.13$ \\
JCR4 (01.02.08) & $52.75^{\circ} \mathrm{S}$ & 19.00 & $58-60 \mathrm{~m}$ & 4.10 & 19.36 & $22.15^{4}$ & 16.15 & $3.28-20.17$ \\
\hline
\end{tabular}

${ }^{1}$ refers to time of the previous day; ${ }^{2}$ phase, waning, $9 \%$ visible; ${ }^{3}$ phase, waxing gibbous, $59 \%$ visible; ${ }^{4}$ phase, waning, $29 \%$ visible.

Table 1b. Environmental parameters for each experimental sample. Experimental conditions in the activity monitor.

\begin{tabular}{lccccc}
\hline $\begin{array}{l}\text { Experiment } \\
\text { (date) }\end{array}$ & Dawn & Dusk & Photoperiod & $\begin{array}{c}\text { Light intensity } \\
\left(\mu \mathrm{mol} \mathrm{m}^{-2} \mathrm{~s}^{1}\right)\end{array}$ & $\begin{array}{c}\text { Water } \\
\text { temperature }\end{array}$ \\
\hline JCR2 $(05.01 .08)$ & 3.00 & 21.30 & $18.5 \mathrm{~L}-5.5 \mathrm{D}$ & 0.0264 & $2.1-2.5^{\circ} \mathrm{C}$ \\
JCR3 $(16.01 .08)$ & 3.00 & 21.00 & $18.0 \mathrm{~L}-6.0 \mathrm{D}$ & 0.0264 & $2.0-2.5^{\circ} \mathrm{C}$ \\
JCR4 (01.02.08) & 4.00 & 20.00 & $18.0 \mathrm{~L}-6.0 \mathrm{D}$ & 0.0264 & $3.2-3.7^{\circ} \mathrm{C}$ \\
\hline
\end{tabular}

high-resolution method of measuring the period of rhythmic fluctuations (Dowse and Ringo 1989; Levine et al. 2002a). MESA and CLEAN are both ways of minimizing the problems encountered with any discretely sampled time series of finite length. MESA operates by fitting an autoregressive model to the data from which the spectrum is calculated, and is equivalent to extending the autocorrelation function in a manner commensurate with 'ignorance' of the data. Sidelobe suppression and resolution are both superior to Fourier analysis alone (Ables 1974; Kay and Marple 1981; reviewed in Levine et al. 2002a). CLEAN has a similar goal in that it reduces the artefacts produced by simple Fourier analysis when dealing with finite discretely sampled time series. It works by assuming there is a 'true, clean' spectrum underlying the actual sampled, 'dirty' one. It takes a 'point source' or expected peak, leaving residuals. These residuals are the 'noise' produced by the limitations of the sampling and analysis systems. This process is iterated until only pure noise remains. In the algorithm, these components can then be used to produce a 'clean spectrum' consisting only of the point sources (Roberts et al. 1987). CLEAN is particularly suitable for processing time series of suboptimal length. To circumvent the latter, in our analysis we added a string of zeros to the data, so as to bring the length of each segment of the experiments to five days, where necessary. We used a Monte-Carlo simulation to calculate the significance of the different rhythmic components identified by CLEAN (Rosato and Kyriakou 2006).

The data sets were analysed using MESA after digital filtering either by removing periodicities equal to or shorter than $4 \mathrm{~h}$ or by applying a two-pole, band-pass Butterworth digital filter to remove both high frequency noise ( $4 \mathrm{~h}$ cut-off) and long-period trends ( $40 \mathrm{~h}$ cut-off). The three highest spectral peaks were reported out from the output (Dowse 2007). To test for the significance of the periodicity, an autocorrela- tion analysis was carried out (Dowse 2007). The rhythmicity index (RI) is a function that assesses the robustness of the estimated rhythmicity, based on the height of the third peak of the autocorrelation analysis. In addition, to ensure that the 12 $\mathrm{h}$ periodicities observed were not artefacts, we took the periods calculated by MESA for the most promising data sets and used those to fit a cosine function using nonlinear regression (Statistical Analysis System, NLIN procedure, Cary, USA) to get values for amplitude, DC offset, and phase. These were then plotted against the original data (see Results).

\section{Results}

Krill are active swimmers, ascending in the water column by continuous movement of their pleopods, and sinking when they are inactive (Kils 1981). In the activity monitor, spatial and temporal variations in locomotor activity of freshlycaught individuals were recorded by two sensors, one at the bottom and one at the top of each channel. Using timer controlled LEDs and neutral density filters we calibrated photoperiod and light intensity to be close to those of the external environment (table 1). As krill are most abundant in the top layers of the ocean during the night, we would expect to measure higher levels of activity with the top sensor at night and higher level of activity with the bottom sensor during the day.

We plotted the amount of activity per $30 \mathrm{~min}$ bin as a function of time, thus obtaining activity profiles. The assessment of individual animals indicated that, under our experimental conditions, E. superba has very noisy locomotor behaviour characterized either by prolonged inactivity interspersed with lasting bursts of locomotion (figure 1) or by complex rhythmicity where more than one periodic component can be found (figure 2). 


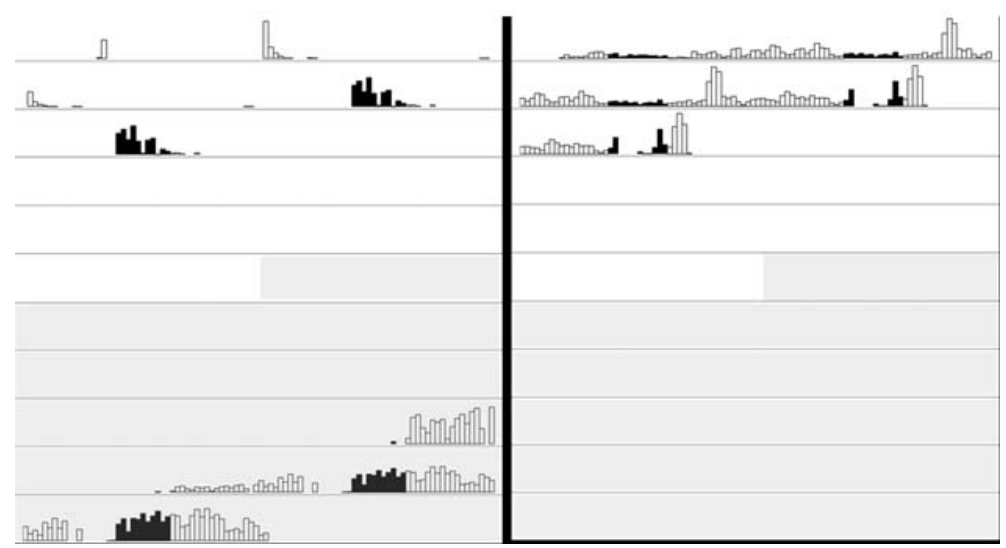

Figure 1. Activity profiles for a single krill over a period of $10+$ days as measured by the bottom (left panel) and the top (right panel) sensor, respectively. Data are double-plotted, namely the top line covers days 1 and 2, the second days 2 and 3, etc. Note that this animal shows sporadic bursts of activity, often prolonged, and that the temporal profiles recorded by the two sensors are very different. The units on the horizontal axis are bins (30 min intervals), those on the vertical axis are amount of activity measured as interruptions of the infrared beam of the relevant sensor. The LD part of the experiment is identified by a white background. The empty vertical bars correspond to activity when the lights are on, the solid black vertical bars correspond to activity when the lights are off. The DD part of the experiment is identified by a grey background. Here the empty bars correspond to activity in the subjective time of lights on, the solid black bars correspond to activity under the subjective time of lights off.

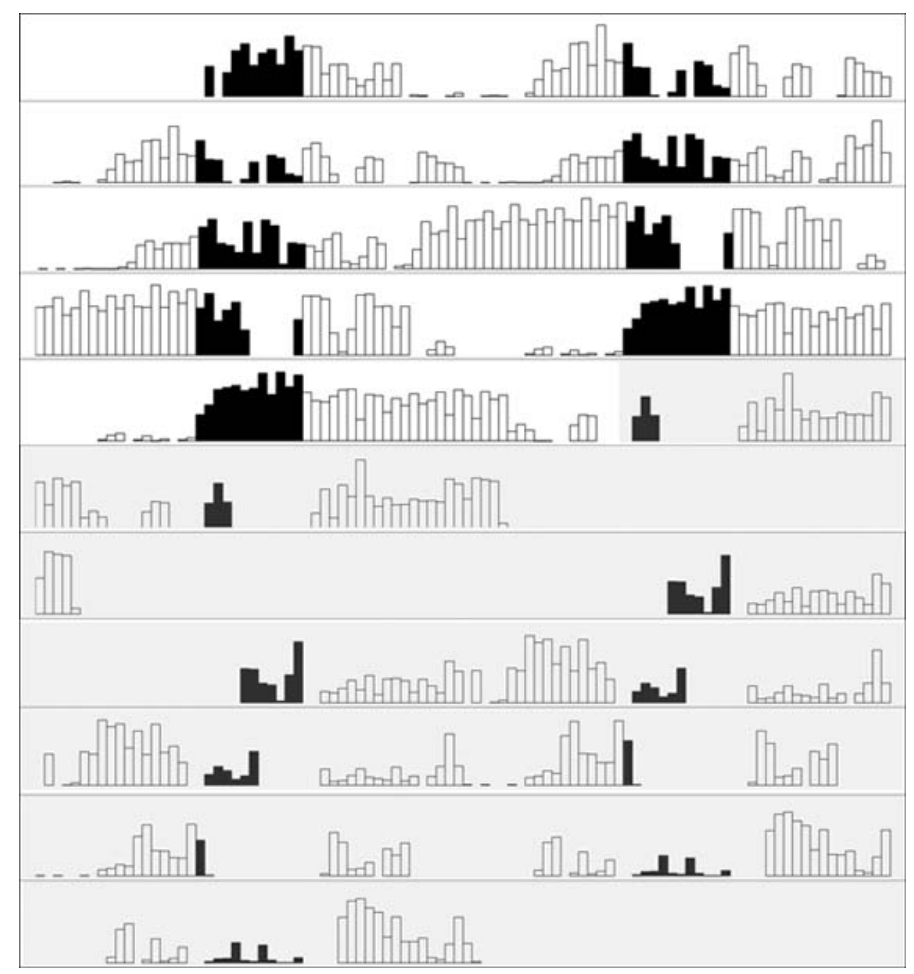

Figure 2. Double-plot of locomotor activity (bottom sensor) for a single krill over a period of $10+$ days. This animal shows three main rhythmic components both in $\operatorname{LD}(\tau=15.2,19.53$ and 25.51, as calculated by CLEAN) and in DD ( $\tau=12.8,19.64$ and 28.83 , as calculated by CLEAN). The same conventions as in figure 1 apply. This krill did not show any activity at the level of the top sensor. 
To look for underlying behavioural rhythms in the data, we first examined the distribution of activity during light (L) and darkness (D) in LD and during subjective lights on (1) and subjective lights off (d) in DD for both the bottom and the top sensors. For each experiment and sensor we calculated the average activity per bin $\left(\mathrm{AA}_{\mathrm{b}}\right)$ in $\mathrm{L}, \mathrm{D}, 1$ and $\mathrm{d}$. We then normalized (for each experiment and sensor) the values of $\mathrm{AA}_{\mathrm{b}}$ for each condition, under $\mathrm{LD}$ or DD-for instance $\left(\mathrm{AA}_{\mathrm{b}} \mathrm{L}\right)_{\text {Norm }}=\mathrm{AA}_{\mathrm{b}} \mathrm{L} /\left(\mathrm{AA}_{\mathrm{b}} \mathrm{L}+\mathrm{AA}_{\mathrm{b}} \mathrm{D}\right)$, and averaged the three experiments.

Figure 3 shows that the mean normalized average amount of locomotor activity per bin is generally higher during the period of actual or subjective lights on for the bottom sensor and during the period of actual or subjective lights off for the top sensor, although significant differences were only observed for the 1 to $d$ (ANOVA, $F_{1,4}=10.532, P<0.05$ ) and the $\mathrm{L}$ to $\mathrm{D}$ (ANOVA, $F_{1,4}=27.779, P<0.01$ ) comparisons for the bottom and top sensors, respectively.

We then measured the period of activity of individual animals. As stated above, in our experiments, Euphausia showed noisy locomotor behaviour. Moreover, several animals did not trigger either the top or the bottom sensor and showed no activity (na). Others had locomotor behaviour that was very sporadic and therefore insufficient to produce any rhythm discernible by the time series analysis programmes (ns). Finally some animals showed rhythmic behaviour (see table 2) and among them a good proportion had rhythms in the circadian range (18-30 h) as calculated by CLEAN and MESA.

The distribution and the median of circadian periods in LD and DD for the bottom and top sensors were found to be different (figure 4). In general, periods were shorter than $24 \mathrm{~h}$ for the bottom sensor and longer than $24 \mathrm{~h}$ for the top sensor. Interestingly, several animals (see figure 5) displaying circadian rhythmicity either in LD or in DD showed two circadian periods for the activity monitored by the same sensor. The median period of locomotor activity in DD increased, compared to LD, for the top sensor and remained shorter than $24 \mathrm{~h}$ for the bottom sensor. In summary, these data suggest that krill display complex circadian periodicity made of two components, one shorter than $24 \mathrm{~h}$, preferentially recorded by the bottom sensor, and one longer than $24 \mathrm{~h}$, preferentially recorded by the top sensor, and show a change of period when moving from entrainment (LD) to constant (DD) conditions. Although very suggestive, the large variability in the data does not allow us to identify a statistically significant difference between sensors or light conditions with this sample size.

Table 2. Proportions of animals in all experiments showing (na) no activity; ns, no significant rhythms; nc, no circadian rhythms; cd, circadian rhythms $18-30 \mathrm{~h}$. Activity is recorded on the bottom (b) or top (t) sensor in the activity monitor, either under a light-dark cycle (LD) or in total darkness (DD).

\begin{tabular}{|c|c|c|c|c|}
\hline \multicolumn{5}{|c|}{ CLEAN analysis } \\
\hline & \multicolumn{2}{|c|}{$\mathrm{LD}$} & \multicolumn{2}{|c|}{ DD } \\
\hline & $\mathrm{b}(\%)$ & $\mathrm{t}(\%)$ & $\mathrm{b}(\%)$ & $\mathrm{t}(\%)$ \\
\hline na & 37 & 40 & 43 & 64 \\
\hline ns & 7 & 10 & 17 & 13 \\
\hline nc & 33 & 23 & 20 & 10 \\
\hline $\mathrm{cd}$ & 23 & 27 & 20 & 13 \\
\hline \multicolumn{5}{|c|}{ MESA analysis } \\
\hline & \multicolumn{2}{|c|}{ LD } & \multicolumn{2}{|c|}{$\mathrm{DD}$} \\
\hline & $\mathrm{b}(\%)$ & $\mathrm{t}(\%)$ & $\mathrm{b}(\%)$ & $\mathrm{t}(\%)$ \\
\hline na & 37 & 40 & 43 & 64 \\
\hline ns & 13 & 13 & 7 & 3 \\
\hline $\mathrm{nc}$ & 23 & 13 & 17 & 13 \\
\hline $\mathrm{cd}$ & 27 & 33 & 33 & 20 \\
\hline
\end{tabular}

The data were also tested using MESA and autocorrelation after applying a band-pass digital filter to remove very short ( $\leq 4 \mathrm{~h})$ and very long $(\geq 40 \mathrm{~h})$ periodicities. This analysis was particularly powerful in testing the significance of ca. $12 \mathrm{~h}(9-15 \mathrm{~h})$ periodic components (evidence of bimodal
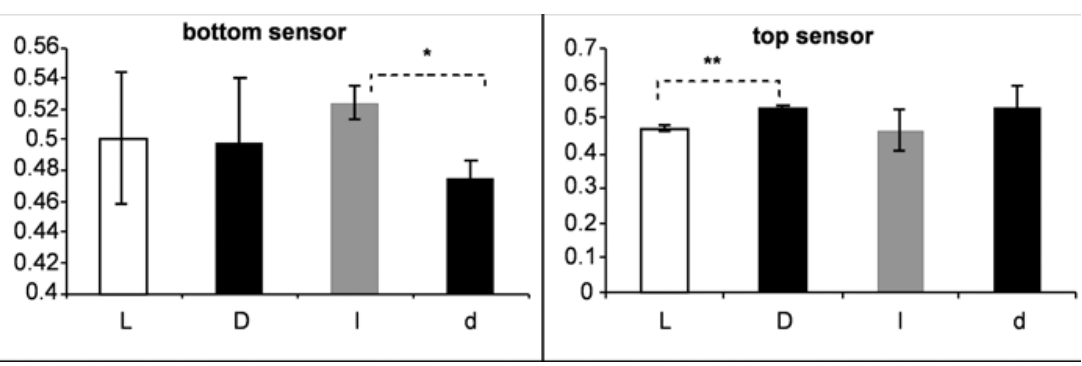

Figure 3. Mean normalized average locomotor activity per bin recorded at the bottom and at the top sensor. All active animals have been included. Activity in light (L) and dark (D) during the LD cycle or in subjective light (l) and subjective dark (d) during the DD cycle is shown. $* P<0.05 ; * * P<0.01$. 

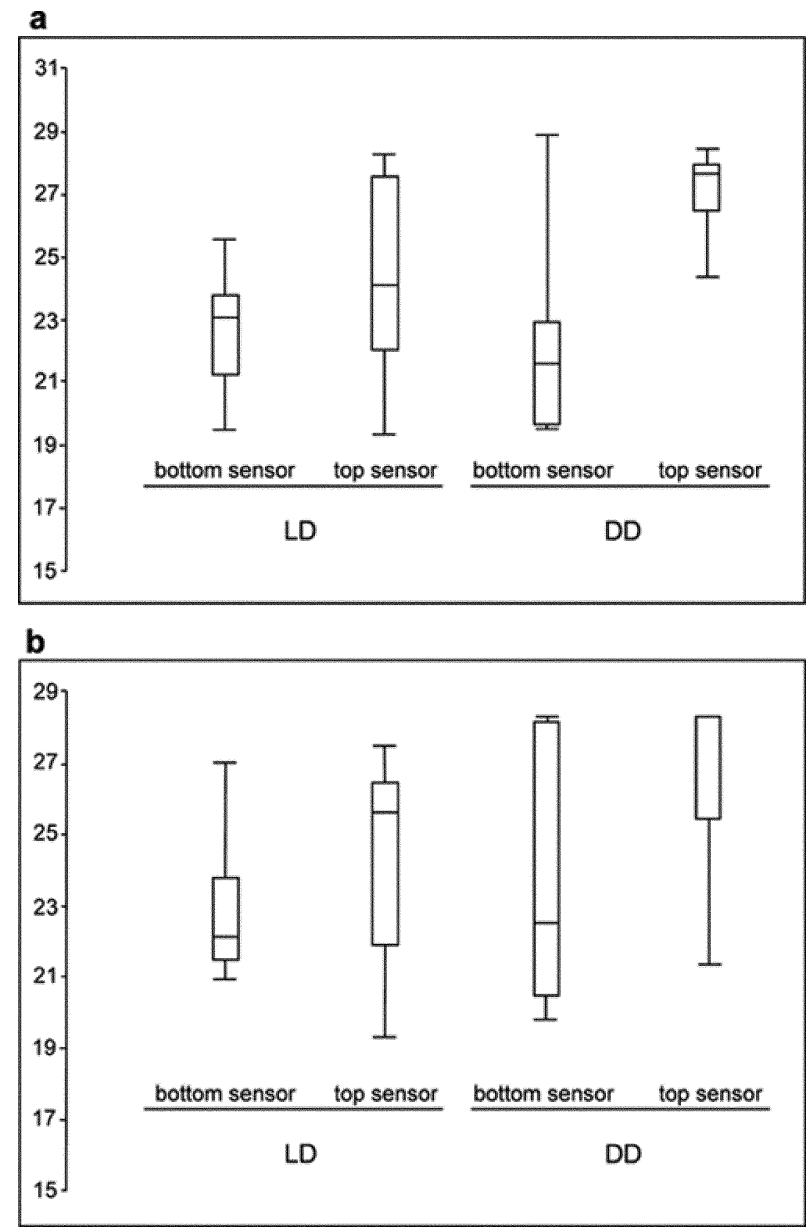

Figure 4. Median (horizontal line) and distribution (the box shows the range of the 25th and 75th percentiles, the whisker shows the 90th percentile) of periodicities (for circadian animals) for bottom and top sensors in LD and DD. (a) Periods calculated by CLEAN. (b) Periods calculated by MESA after removal of short trends $(\leq 4$ h).

circadian rhythms) found in the activity of many animals both in LD and in DD and for both sensors. Figure 6 depicts two records which show clear bimodal circadian periodicity. Here the data have been band-pass filtered, and the sinusoid, derived from the data using the period $(\tau)$ computed after MESA and autocorrelation analysis, is superimposed (see Materials and methods). Figure 6,a shows a moderately strong example of an animal in LD. The RI is 0.07 and the computed period is $10.4 \mathrm{~h}$. It is unlikely that this rhythmicity is artifactual as the MESA and autocorrelation algorithms are very distant in methodology. It is also clear that the sinusoid fits the data well, and that period and phase remain constant throughout the record. Similarly, Figure 6,b shows an animal in DD. Here, the rhythmicity is quite robust with $\tau=12.6$ $\mathrm{h}$ and $\mathrm{RI}=0.36$, which is very strong. The fitted sinusoid is also strong and the animal's activity remains constant in phase, period, and amplitude throughout.
In addition to the circadian and bimodal circadian periodicities, MESA and autocorrelation identified multiple examples of ultradian rhythms. For instance, figure 7 shows an animal that is quite powerfully rhythmic in a high-frequency mode $(\tau=5.23 \mathrm{~h}, \mathrm{RI}=0.18)$ with a sinusoid fitted as above.

\section{Discussion}

The most striking behaviour of many pelagic animals is a DVM from the inner ocean to the surface layers at the beginning of the night and vice versa at the beginning of the day. The widespread distribution of this behaviour across taxa suggests it probably confers a selective advantage by balancing two conflicting needs, food/exposure and shelter/starvation, through an optimal temporal allocation of the two behaviours. However, there is evidence that DVM patterns are not always simple. For some species, the population can be divide into deep and shallow layers at the same time (Frost 1988) suggesting that more than one cycle of DVM occurs per day and that light is not an overwhelming trigger for sinking. It follows that, for these species, synchronisation of DVM across individuals is not always achieved and that light:dark is not the main environmental cycle (or Zeitgeber) driving the rhythmic behaviour of the organisms.

Antarctic krill has such a complex DVM pattern. Acoustic data suggest that the DVM of E. superba shows a main $24 \mathrm{~h}$ component (night up, day down) and a smaller $12 \mathrm{~h}$ component (ascending again in the middle of the day). The latter is negligible in conditions of high food availability but becomes progressively more important as the concentration of food declines (Godlewska 1996). Moreover, even under conditions of food abundance, the DVM dynamics might be more complex than assessed at the population level, with individuals swimming to the surface to eat, sinking when full and migrating up again when digestion is complete, with an estimate of up to three cycles per night (Tarling and Johnson 2006). Behavioural observations of pelagic individuals in natural conditions have rarely been achieved and are not easy to accomplish. Thus the study of behavioural patterns of krill in a laboratory setting is important as it can help the interpretation of data from the field and perhaps even explain some of the variability in the reports of DVM for different swarms (Hardy and Gunther 1935; Everson 1983). In this study, we undertook a laboratory investigation of the circadian regulation of locomotor activity under entrainment (LD) and freerun (DD) conditions at the individual level. The long term aim was to understand how the circadian clock might contribute to the DVM, a behaviour usually described as having a strong environmental component (Ringelberg 1995; Pearre 2003).

We used a custom-built activity monitor where upright cylindrical tubes ( $48 \mathrm{~cm}$ in length) filled with 51 of seawater constituted a channel monitored by infrared sensors located $5 \mathrm{~cm}$ from the bottom and $5 \mathrm{~cm}$ from the top of the tube. 


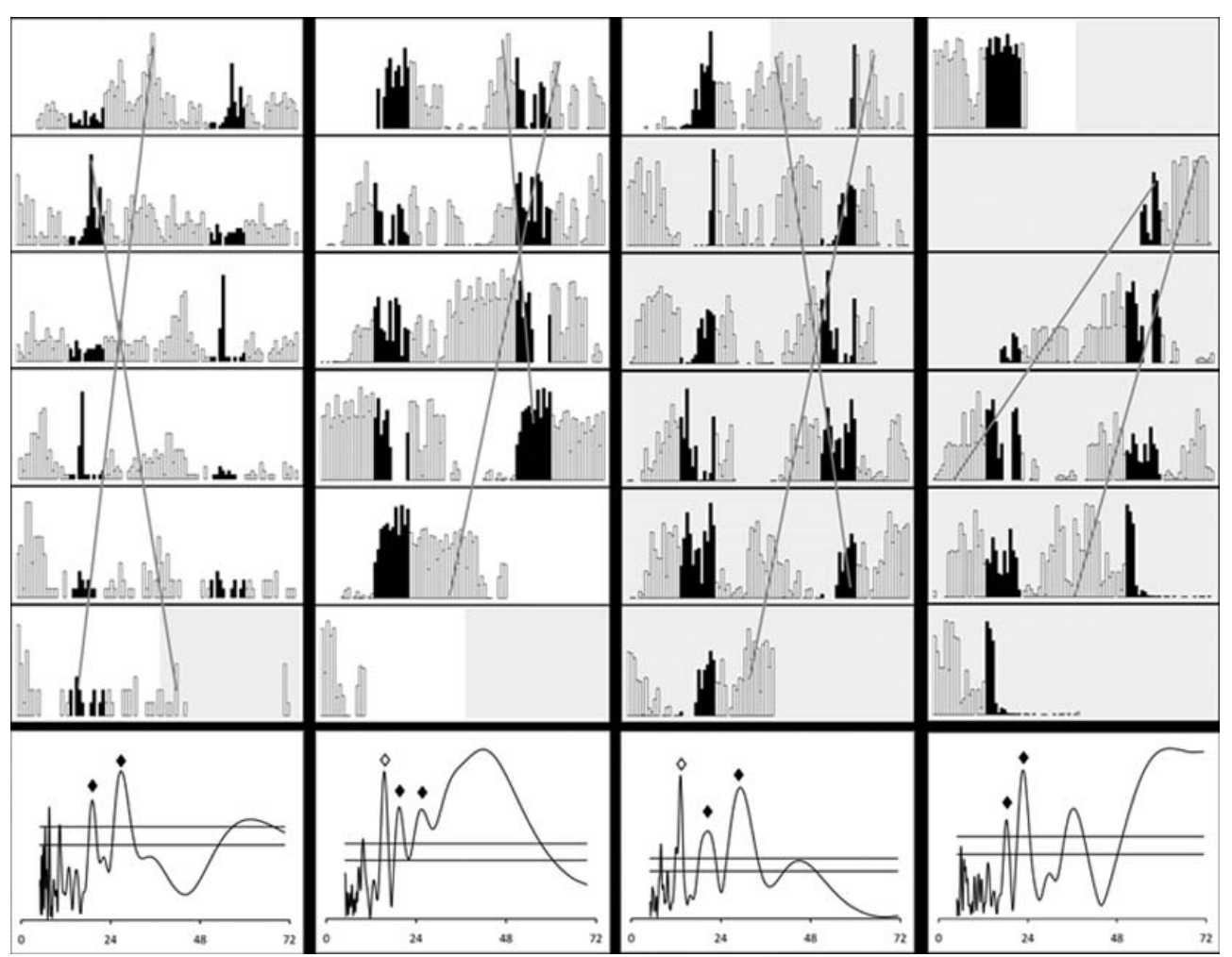

Figure 5. Example of activity records showing multiple rhythmicity. Double plots of LD (a and b) and DD (c and d) activity are as in figure 1. Grey lines have been superimposed to help in visualizing the circadian periods. The graph at the bottom of each activity record shows the output of CLEAN. Each peak corresponds to a period (whose value is given in hour on the $\mathrm{X}$-axis. The values on the Y-axis correspond to an arbitrary scale). A peak is deemed significant if it crosses the top horizontal line representing the $99 \%$ confidence limit. The full diamonds highlight significant circadian peaks, the open diamonds show significant ca $12 \mathrm{~h}$ periods. (a) JCR2, LD, 11t. (b) JCR4, LD, 6b. (c) JCR4, DD, 1b. (d) JCR4, DD, 2b.
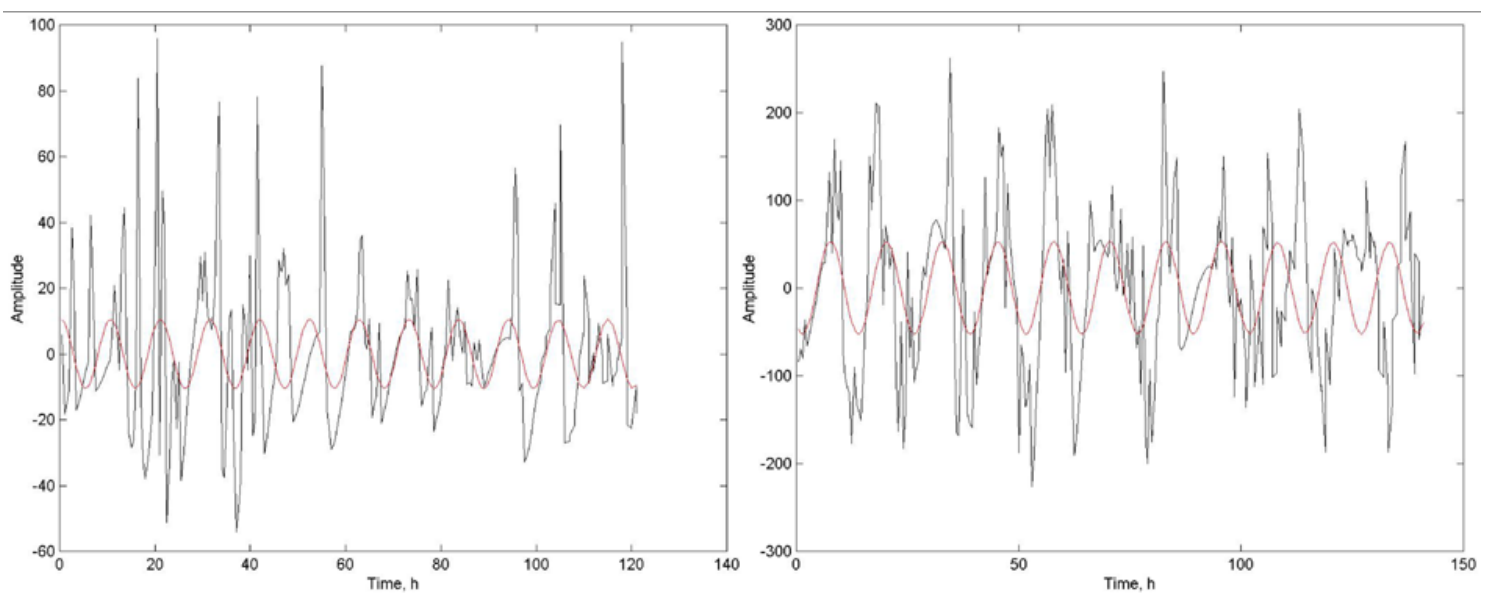

Figure 6. Circa 12-h rhythms in LD (a) and in DD (b). Band-pass filtered locomotor activity data are shown as amplitude as a function of time with a superimposed sinusoid derived from the data using the computed $\tau$ (after MESA and autocorrelation analysis). (a) Top sensor activity record in LD for an individual from JCR2 showing a period of $10.4 \mathrm{~h}$. (b) Bottom sensor activity record in DD for an individual from JCR2 showing a period of $12.6 \mathrm{~h}$. 


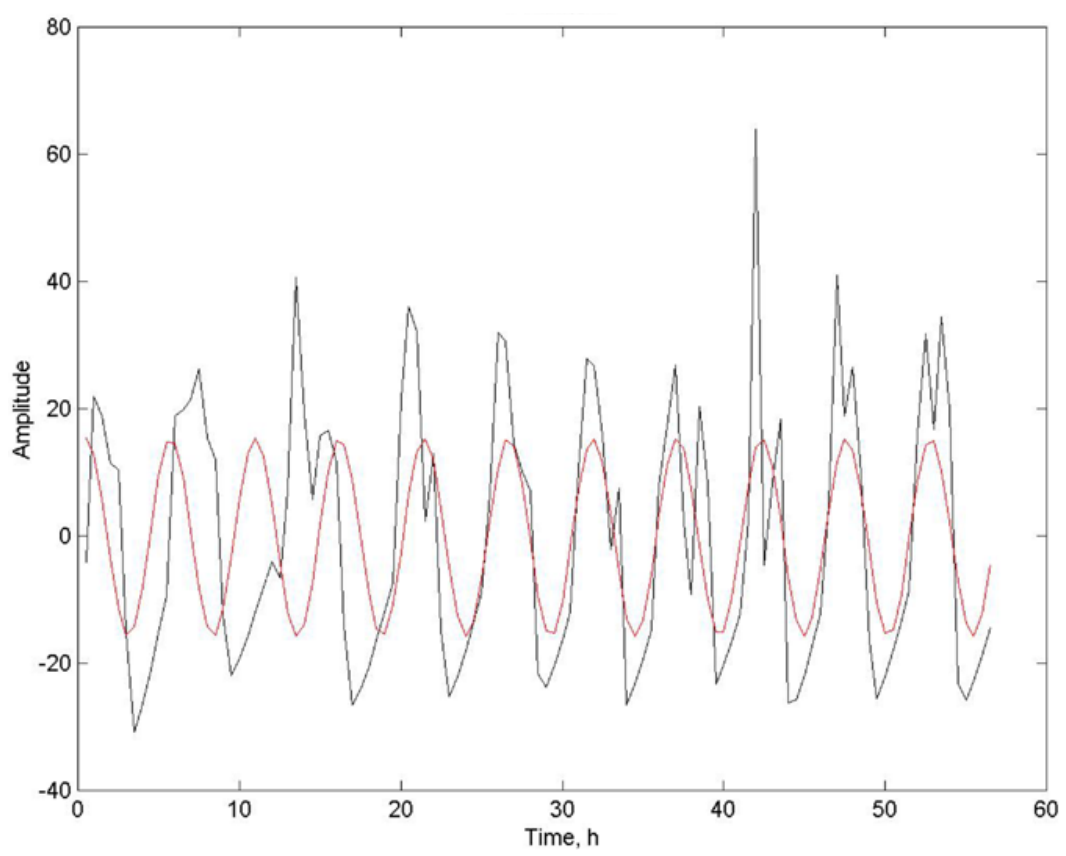

Figure 7. Bottom sensor activity record in DD for an individual from JCR2 showing an ultradian period of $5.23 \mathrm{~h}$. Data are reported as in figure 6.

Krill were maintained for five days in LD and for 3-5 days in DD with no food. Under these conditions $50 \%$ (LD) to $70 \%$ (DD) of the animals were not active, at least not enough to generate a rhythmic pattern, suggesting that damage during capture and sorting or lack of food might have affected locomotor activity in these very active animals. Nevertheless $50-60 \%$ of rhythmic krill showed a circadian period, although we cannot rule out that these animals might also have been influenced by the factors detailed above. The design of the activity monitor was based upon the assumption that light is the main Zeitgeber regulating DVM, therefore, mimicking the DVM, the bottom and the top sensors would be preferentially triggered during lights on and lights off, respectively. In our experiments the periods of lights on (actual or subjective) were three times longer than those of lights off (actual or subjective), thus a simple comparison between light (actual or subjective) and dark (actual or subjective) activity would have been biased. Instead, we compared the average activity per bin for lights on (actual or subjective) and lights off (actual or subjective) after normalization to the total activity across the whole experiment and we calculated the mean value for the three experiments we conducted. We were able to show that the mean activity per bin is generally higher at the time of lights off (or expected lights off) for the top sensor and during the time of light on (or expected lights on) for the bottom sensor, with some of the comparisons being statistically significant (see figure 3). For instance, the mean activity per bin measured by the bottom sensor was significantly higher during the subjective lights on than the subjective lights off. Thus, a different rate of activity is maintained under constant conditions suggesting that an endogenous circadian clock rather than the LD cycle might be its cause.

We then used spectral analysis (CLEAN and MESA) to calculate the period of locomotor activity either without manipulation of the data (CLEAN) or after applying a digital filter to remove short $(\leq 4 \mathrm{~h})$ trends. We found that krill has complex rhythms made of two circadian components, one shorter than $24 \mathrm{~h}$, preferentially recorded by the bottom sensor, and one longer than $24 \mathrm{~h}$, preferentially recorded by the top sensor, although the large variability and the small sample size did not allow us to identify a statistically significant difference between sensors or light conditions. It is noteworthy that even under LD, krill did not display a robust $24 \mathrm{~h}$ rhythm (a median value of ca. $24 \mathrm{~h}$ is shown only by CLEAN for the top sensor), which is what would be expected if the animals were fully entraining to the light:dark cycle. In our experiments, light intensity, photoperiod and temperature were kept within the normal range experienced by krill during summer, thus our interpretation is that the inability to entrain is not due to nonphysiological light or temperature conditions, but to some other factor. This would suggest that for krill the LD cycle may not be such a dominant Zeitgeber as it is for some other animals. Although the above result seems in agreement with Pittendrigh et al. (1991) theory of reduced light sensitivity of the clock for organisms adapted to high latitudes, the total lack of entrainment in our experiments suggests that one or more environmental variables absent in our set-up are likely necessary for entrainment to occur. On the basis of the ecology of krill, it is reasonable to suggest that natural rhythmic activities (absent in our exper- 
imental conditions) such as feeding (Godlewska 1996) and social interactions (Everson 1982, 1983) might be really important for rhythmic behaviour. The latter might be particular important as krill disperse at night and aggregate in swarms during the day (Everson 1982, 1983). Clearly, a social strategy based on rhythms of dispersion/aggregation that are synchronised to the LD cycle is optimal, reducing competition for food during the night and enhancing the chances of survival for the individual (i.e., security in numbers) during the day. Social influences on rhythmicity are not unprecedented as they have been demonstrated in a wide variety of animals, including Drosophila in which the circadian clock can be reset by olfactory cues (Levine et al. 2002b). Antarctic krill are reported to be unresponsive to external stimuli when isolated in laboratory aquaria and only exhibit their normal schooling behaviour when presented with appropriate environmental conditions (Strand and Hamner 1990). Perhaps pressure might also contribute as a Zeitgeber in Antarctic krill, since the animals experience considerable variation in hydrostatic pressure during their daily vertical movements. It has been shown that crustaceans are able to detect changes in pressure (Fraser and Macdonald 1994) and that these changes can affect their locomotor activity and behaviour (Macdonald 1997). However, the effects of pressure on activity in Antarctic krill have not been investigated and it was not possible to incorporate any pressure variation into the present experimental arrangement. In conclusion, we suggest that for krill the principal Zeitgeber might not be the LD cycles, as for more common model systems such as flies and mammals, but rather the combined cycles of LD, social interactions and, perhaps, pressure. This hypothesis, eventually testable, might explain why the LD cycle alone, usually the strongest Zeitgeber, is not able to entrain individual krill under our experimental conditions.

We also suspect that the unnatural absence of those environmental variables might be responsible for the multiple rhythms we observed. We identified different periods for the two sensors in animals that triggered both, but also we found multiple rhythms in some individuals whose activity was recorded only at the top or the bottom of the channel. Multiple rhythms or 'splitting' have previously been described for several organisms and can be elicited by unnatural laboratory conditions. For instance in the fruit fly D. melanogaster splitting can be triggered by constant light (LL) especially in combination with mutations affecting the circadian photopigment cryptochrome (Yoshii et al. 2004; Rieger et al. 2006). In rats, splitting can be forced by non $24 \mathrm{~h} \mathrm{LD}$ cycles (de la Iglesia et al. 2004). The simplest explanation for this unusual behaviour is that the circadian pacemakers (controlling locomotor activity) of flies and rats are made of multiple oscillators (clock neurons) showing a different response to these peculiar light conditions and thus becoming uncoupled. Depending upon the experimental conditions, these oscillators either free run showing their different endogenous periods (one shorter, the other longer than $24 \mathrm{~h}$, as for the Drosophila example) or show an entrained component (with the same period as the LD cycle) and an endogenous period of more than $24 \mathrm{~h}$ (as observed in rats). In our experiments we used light and temperature compatible with the environmental conditions at the time of capture. However, an unnatural lack of food (under a summer photoperiod), of pressure and of social interactions might have triggered the splitting, revealing different circadian neurons running, under these conditions, with different periods.

To add to the complexity of the locomotor activity patterns of krill, we identified a ca. $12 \mathrm{~h}$ rhythmic component, for a high proportion of individuals (ca. $68 \%$ of rhythmic animals). This was particularly evident after further filtering of the data to remove both short $(\leq 4 \mathrm{~h})$ and long $(\geq 40$ h) trends, and performing MESA and autocorrelation analysis. Endogenous rhythms of around $12 \mathrm{~h}$ have often been described in crustaceans, mainly those dwelling in the intertidal zone (e.g. Naylor 1958). However animals like krill, living beyond the influence of tidal movements might also show evidence of 12-h rhythms. For instance periodogram analysis of locomotor activity in the Norway lobster (Nephrops norvegicus) under constant dark conditions has revealed the presence of both 24-h and 12-h rhythms (Aguzzi et al. 2004). We interpret the ca. 12-h rhythms in our data as evidence of bimodality of the circadian-behavioural output. These periodicities form a cluster around $12 \mathrm{~h}$, unlike real ultradians that are scattered all over the spectrum (Dowse 2008). This interpretation fits well with observations in the natural environment of a $12 \mathrm{~h}$ component in DMV periodicity becoming predominant when food is scarce (Godlewska 1996). We also have identified ultradian rhythms in our experiments and these can be quite strong, rivalling the $24 / 12 \mathrm{~h}$ periodicity in clarity. It has been suggested that high frequency rhythms that appear when the circadian (Dowse and Ringo 1987) or tidal (Dowse and Palmer 1990) clock is weak or disrupted in some way may be evidence of even higher-frequency oscillators that underlie the circadian clock (Dowse 2008).

In conclusion, our original question was whether or not there is a demonstrable effect of the circadian clock on the DVM of E. superba. We have identified endogenous rhythms of locomotor activity with a circadian period in several individuals maintained in a laboratory setting. Several more individuals showed a ca. $12 \mathrm{~h}$ period which we interpret, on the basis of experimental data from the natural environment (Godlewska 1996), as evidence of a circadian bimodal output in their behaviour. Our experimental conditions were unnatural because of the lack of food, social interactions and changes in hydrostatic pressure, which probably influenced the behavioural output. However, our results demonstrate that, in these organisms, modulation of locomotor behaviour by the circadian clock is possible, which supports a role for the clock in DMV.

Our experiments have offered a glimpse of a multiple oscillator system in krill. At present, we do not know anything about the nature and the organization of clock neurons 
forming the behavioural pacemaker of this crustacean. We speculate that a plastic multi-oscillator system responsive to several environmental variables might be advantageous for an organism that extends into regions which endure 24-h periods of continuous light or dark. In fact, by varying the contribution of the different neurons to the overt behavioural rhythm, the pacemaker would be able to fine-tune the endogenous period and the temporal allocation of activity/rest episodes during the day to best suit the condition of the organism in the environment. Taken to the extreme, when animals have to face dramatic changes in LD that vary from DD to LL, the decoupling of the oscillators would result in annihilation of the rhythmic behavioural output, which would not be relevant and may be potentially detrimental in prolonged DD or LL (van Oort et al. 2005). It would, however, keep the circadian clock ticking in different neurons which might be important for physiological or metabolic reasons (i.e. to keep the difference in phase in the activity of different organs, or to separate temporally competing enzymatic reactions occurring in the same cells). Krill maintain other longer-term rhythms such as the moulting and reproductive cycles that happen over increments of 10 or more days (Tarling et al. 2007). There have been a number of instances where such cycles appear to be synchronized within krill swarms (Buchholz et al. 1996) which suggests there is interplay of internal clocks and exogenous cues coordinating these process. Further, spawning is known to occur mainly between 20:00 h and 04:00 h (Bargmann 1937; Tarling et al. 1999), which indicates there are even daily rhythms associated with physiological functions independent of DVM and feeding. Our current efforts to identify circadian clock genes and to describe their temporal and spatial patterns of expression will provide powerful tools for a better description of the circadian clock of krill in the near future.

\section{Acknowledgements}

This work was supported by an Antarctic Funding Initiative grant from the Natural Environment Research Council, UK. The help and co-operation of the officers and crew of the RRV 'James Clark Ross' and the participants of cruise JR177 are gratefully acknowledged. We would like to thank the School of Biological Sciences Workshop at the University of Leicester for making the activity monitor.

\section{References}

Ables J. G. 1974 Maximum entropy spectral analysis. Astron. Astrophys. Suppl. Ser. 15, 383-393.

Aguzzi J., Company J. B. and Abello P. 2004 Locomotor activity rhythms of continental slope Nephrops norvegicus (Decapoda: Nephropidae). J. Crustacean Biol. 24, 282-290.

Bargmann H. E. 1937 The reproductive system of Euphausia superba. Discovery Rep. 14, 327-349.

Buchholz F., Watkins J. L., Priddle J., Morris D. J. and Ricketts C. 1996 Moult in relation to some aspects of reproduction and growth in swarms of Antarctic krill, Euphausia superba. Mar. Biol. 127, 201-208.
Chatfield C. 1989 The analysis of time series: an introduction. Chapman and Hall, London.

Cottier F. R., Tarling G. A., Wold G. A. and Falk Petersen S. 2006 Unsynchronized and synchronized vertical migration of zooplankton in a high arctic fjord. Limnol. Oceanogr. 51, 25862599.

de la Iglesia H.O., Cambras T., Schwartz W. J. and Diez-Noguera A. 2004 Forced desynchronization of dual circadian oscillators within the rat suprachiasmatic nucleus. Curr. Biol. 14, 796-800.

Dowse H. B. 2007 Statistical analysis of biological rhythm data. In Circadian rhythms: methods and protocols (ed. E. Rosato), pp. 29-48. Humana Press, Totowa.

Dowse H. B. 2008 Mid-range ultradian rhythms in Drosophila and the circadian clock problem. In Ultradian rhythmicity in biological systems: an inquiry into fundamental principles (ed. D.L. Lloyd and E. Rossi). Springer, Berlin (in Press).

Dowse H. B. and Ringo J. 1987 Further evidence that the circadian clock in Drosophila is a population of coupled ultradian oscillators. J. Biol. Rhythms 2, 65-76.

Dowse H. B. and Ringo J. M. 1989 The search for hidden periodicities in biological time series revisited. J. Theor. Biol. 139, 487-515.

Dowse H. B. and Palmer J. D. 1990 Evidence for ultradian rhythmicity in an intertidal crab. In Progress in clinical and biological research, Vol. 341B. Chronobiology: its role in clinical medicine, general biology and agriculture, Part B (ed. D. K. Hayes, R. Pauly and R. Reiter), pp. 691-697. Wiley Liss, New York.

Everson I. 1982 Diurnal variations in mean volume backscattering strength of an Antarctic krill (Euphausia superba) patch. $J$. Plank. Res. 4, 155-162.

Everson I. 1983 Variations in vertical distribution and density of krill swarms in the vicinity of South Georgia. Mem. Natl. Inst. Polar Res. 27, 84-92.

Fernández de Miguel F. and Aréchiga H. 1994 Circadian locomotor activity and its entrainment by food in the crayfish Procambarus clarkii. J. Exp. Biol. 190, 9-21.

Fraser P. J. and Macdonald A. G. 1994 Crab hydrostatic pressure sensors. Nature 371, 383-384.

Frost B. W. 1988 Variability and possible adaptive significance of diel vertical migration in Calanus pacificus, a planktonic marine copepod. Bull. Mar. Sci. 43, 675-694.

Forward R. B. 1988 Diel vertical migration: zooplankton photobiology and behaviour. Oceanogr. Mar. Biol. Annu. Rev. 26, 361393.

Gliwicz J. Z. 1986 Predation and the evolution of vertical migration in zooplankton. Nature 320, 746-747.

Godlewska M. 1996 Vertical migrations of krill (Euphausia superba Dana). Polish Arch. Hydrobiol. 43, 9-63.

Hardy A. C. and Gunther E. R. 1935 The plankton of the South Georgia whaling grounds and adjacent waters, 1926-1927. Discovery Rep. 11, 1-456.

Kay S. M. and Marple S. G. Jr 1981 Spectrum analysis, a modern perspective. IEEE Proc. 69, 1380-1419.

Kils U. 1981 The swimming behaviour, swimming performance and energy balance of Antarctic krill, Euphausia superba. BIOMASS Scient. Res. Ser. 3, 1-121.

Levine J. D., Funes P., Dowse H. B. and Hall J. C. 2002a Signal analysis of behavioural and molecular cycles. BMC Neurosci. 3, $1-25$.

Levine J. D., Funes P., Dowse H. B. and Hall J. C. 2002b Resetting the circadian clock by social experience in Drosophila melanogaster. Science 298, 2010-2012.

Macdonald A. G. 1997 Hydrostatic pressure as an environmental factor in life processes. Comp. Biochem. Phys. A 116, 291-297. 
Mauchline J. and Fisher L. R. 1969 The biology of euphausiids. Adv. Mar. Biol. 7, 1-454.

Naylor E. 1958 Tidal and diurnal rhythms of locomotor activity in Carcinus maenas. J. Exp. Biol. 35, 602-610.

Pearre S. Jr 2003 Eat and run? The hunger/satiation hypothesis in vertical migration: history, evidence and consequences. Biol. Rev. 78, 1-79.

Pittendrigh C. S., Kyner W. T. and Takamura T. 1991 The amplitude of circadian oscillations: temperature dependence, latitudinal clines, and the photoperiodic time measurement. J. Biol. Rhythms 6, 299-313.

Rieger D., Shafer O. T., Tomioka K. and Helfrich-Forster C. 2006 Functional analysis of circadian pacemaker neurons in Drosophila melanogaster. J. Neurosci. 26, 2531-2543.

Ringelberg J. 1995 Changes in light intensity and diel vertical migration: a comparison of marine and freshwater environments. J. Mar. Biol. Assoc. UK 75, 15-25.

Roberts D. H., Lehár J. and Dreher J. 1987 Time series analysis with CLEAN. I. Derivation of a spectrum. Astron. J. 93, 968-989.

Rosato E. and Kyriakou C. P. 2006 Analysis of locomotor activity rhythms in Drosophila. Nat. Protoc. 1, 559-568.

Strand S. W. and Hamner W. M. 1990 Schooling behavior of Antarctic krill (Euphausia superba) in laboratory aquaria: reactions to chemical and visual stimuli. Mar. Biol. 106, 355-359.
Tarling G. A. and Johnson M. L. 2006 Satiation gives krill that sinking feeling. Curr. Biol. 16, R173-R175.

Tarling G. A., Cuzin-Roudy J. and Buchholz F. 1999 Vertical migration behaviour in the northern krill Meganyctiphanes norvegica is influenced by moult and reproductive processes. Mar. Ecol. Prog. Ser. 190, 253-262.

Tarling G. A., Cuzin-Roudy J., Thorpe S., Shreeve R., Ward P. and Murphy E. 2007 Recruitment of Antarctic krill Euphausia superba in the South Georgia region: adult fecundity and the fate of larvae. Mar. Ecol. Prog. Ser. 331, 161-179.

van Oort B. E. H., Tyler N. J. C., Gerkema M. P., Folkow L., Schytte-Blix A. and Stokkan K. A. 2005 Circadian organization in reindeer. Nature 438, 1095-1096.

Velsch J.-P. and Champalbert G. 1994 Swimming activity rhythms in Meganyctiphanes norvegica. C. R. Acad. Sci. Paris Sci. Vie 317, 857-862.

Yoshii T., Funada Y., Ibuki-Ishibashi T., Matsumoto A., Tanimura T. and Tomioka K. 2004 Drosophila cryb mutation reveals two circadian clocks that drive locomotor rhythm and have different responsiveness to light. J. Insect Physiol. 50, 479-488.

Youngbluth M. J. 1975 The vertical distribution and diel migration of euphausiids in the central waters of the Eastern South Pacific. Deep-Sea Res. 22, 519-536. 